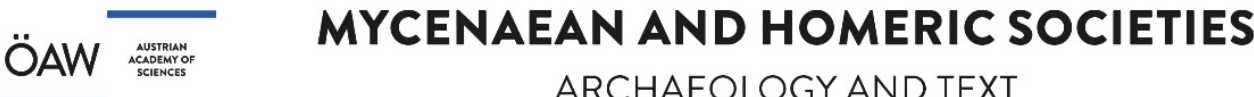 \\ ARCHAEOLOGY AND TEXT
}

Collected Papers of

\section{SIGRID DEGER-JALKOTZY}

edited by Birgitta Eder and Ulrike Schuh

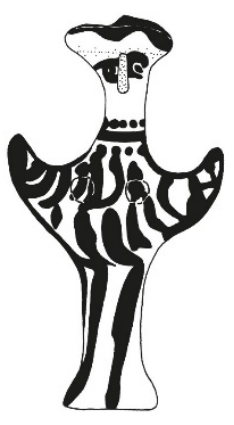

14.

S. Deger-Jalkotzy

\section{Landbesitz und Sozialstruktur im mykenischen Staat von Pylos}

in: M. Heltzer - E. Lipiński (eds.), Society and Economy in the Eastern Mediterranean (c. 1500-1000 B.C.). Proceedings of the International Symposium held at the University of Haifa from the $28^{\text {th }}$ of April to the $2^{\text {nd }}$ of May 1985, Orientalia Lovaniensia Analecta 23 (Leuven 1988) 31-52.

(c) Peeters

mit freundlicher Genehmigung / with kind permission

Dieses Dokument darf ausschließlich für wissenschaftliche Zwecke genutzt werden (Lizenz CC BY-NC-ND), gewerbliche Nutzung wird urheberrechtlich verfolgt. 
ORIENTALIA LOVANIENSIA

ANALECTA

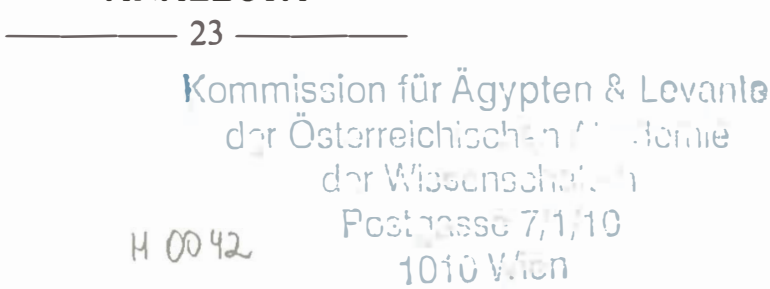

\section{SOCIETY AND ECONOMY \\ IN THE EASTERN MEDITERRANEAN \\ (c. 1500-1000 B.C.)}

\section{Proceedings of the International Symposium \\ held at the University of Haifa}

from the 28th of April to the 2nd of May 1985

\section{EDITED}

BY

M. HELTZER and E. LIPIŃSKI

UITGEVERIJ PEETERS

LEUVEN

1988

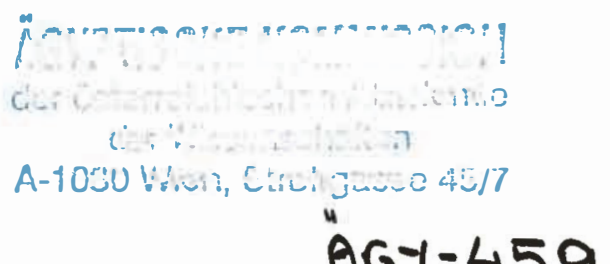


Editorial Board of this Proceedings Volume

Prof. M. Dothan (Univ. of Haifa), Prof. M. Heltzer (Univ. of Haifa),

Prof. E. Lipiński (K. Univ. Leuven), Prof. M. Weinfeld (Hebrew Univ. of Jerusalem).

(C) by Departement Oriëntalistiek

Orientalia Lovaniensia Analecta

Blijde Inkomststraat 21, B-3000 Leuven/Louvain (Belgium)

All rights reserved, including the rights to translate or to reproduce this book or parts thereof in any form.

D/1988/0602/48

ISBN 90-6831-135-2

Printed in Belgium by Orientaliste, Leuven. 


\section{CONTENTS}

FOREWORD. . . . . . . . . . . . . . . . . . . VII

ABbREVIATIONS . . . . . . . . . . . . . . . IX

Preface by Prof. Uriel Rappaport, Rector of the University of Haifa XIII

B. MAZAR, Introduction . . . . . . . . . . . . . . 1

M. Heltzer, The Late Bronze Age Service System and Its Decline 7

A. UchITEL, The Archives of Mycenaean Greece and the Ancient Near East . . . . . . . . . . . . . . . . . . 19

S. DeGER-JALKotZY, Landbesitz und Sozialstruktur im mykenischen Staat von Pylos . . . . . . . . . . . . . . . 31

S. HILler, Dependent Personnel in Mycenaean Texts. . . . . 53

R. HAASE, Some Problems of Hittite Law and Jurisdiction . . . 69

Sh. ARbeli, The Removal of the Tawananna from Her Position . 79

Z. Ben-BaraK, The Legal Status of the Daughter as Heir in Nuzi and Emar . . . . . . . . . . . . . . . . . . . 87

E. GaÁL, The Social Structure of Alalah . . . . . . . . . . 99

P. VARGYAS, Stratification sociale à Ugarit . . . . . . . . 111

E. LIPIŃSKI, The Socio-Economic Condition of the Clergy in the Kingdom of Ugarit . . . . . . . . . . . . . . 125

A. SKaIST, $A$ Unique Closing Formula in the Contracts from Ugarit 151

Sh. IZRE'EL, When was the "General's Letter" from Ugarit written? 160

N. Na'Aman, Pharaonic Lands in the Jezreel Valley in the Late Bronze Age. . . . . . . . . . . . . . . . . 177

H. G. Buchноцz, Der Metallhandel des zweiten Jahrtausends im Mittelmeer. . . . . . . . . . . . . . . . . 187

A. Altman, Trade between the Aegean and the Levant in the Late Bronze Age: Some Neglected Questions . . . . . . . . 229

I. Singer, The Origin of the Sea Peoples and Their Settlement on the Coast of Canaan. . . . . . . . . . . . . . . . 239

A. Mazar, Some Aspects of the "Sea Peoples"' Settlement . . 251

A. Raban, The Constructive Maritime Role of the Sea Peoples in the Levant . . . . . . . . . . . . . . . . . 261

M. Dothan, The Significance of Some Artisans' Workshops along the Canaanite Coast. . . . . . . . . . . . . . 295

J. BaLensi, Tell Abū Hawām: un cas exceptionnel? . . . . . 305 
D. Eitam, The Settlement of Nomadic Tribes in the Negeb Highlands during the 11th Century B.C.

A. ZERTAL, The Water Factor during the Israelite Settlement Process in Canaan . . . . . . . . . . . . . . 341

M. Weinfeld, The Promise to the Patriarchs and Its Realization: An Analysis of Foundation Stories . . . . . . . . . . 353 General Discussion . . . . . . . . . . . . . . . . 371

Indexes by M. Heltzer and A. Shockron-CASPI . . . . . 383

1. Personal Names . . . . . . . . . . . . . . 383

2. Ethnic and Geographic Names . . . . . . . . . 387

3. Divine Names . . . . . . . . . . . . . . . 394

4. Terms of social and economic significance . . . . . . 394

1. Akkadian . . . . . . . . . . . . . . . 394

2. Egyptian . . . . . . . . . . . . . 395

3. Greek . . . . . . . . . . . . . . . . 395

4. Linear B . . . . . . . . . . . . . . . . 395

5. Hebrew . . . . . . . . . . . . . . 396

6. Hittite . . . . . . . . . . . . . 396

7. Sumerian and Sumerograms . . . . . . . . 396

8. Ugaritic . . . . . . . . . . . . . . . 396 


\title{
LANDBESITZ UND SOZIALSTRUKTUR IM MYKENISCHEN STAAT VON PYLOS
}

\author{
SIGRID DEGER-JALKOTZY
}

Verglichen mit den reichen literarischen Quellen der Kulturen des Alten Orients, bieten die mykenischen Linear B-Texte eine nur schmale Materialbasis für die Lebensverhältnisse im mykenischen Griechenland. Ihre Zahl ist nicht groß ${ }^{1}$ und ihre Aussagen sind in vieler Hinsicht begrenzt ${ }^{2}$. Es überrascht daher nicht, daß ihre Interpretationen oft divergieren, daß es kontroversielle Auffassungen über ihren Inhalt gibt, und da $ß$ nur in wenigen Fällen so etwas wie eine communis opinio erzielt werden konnte. Dies betrifft vor allem die Bemühungen um die Erforschung der mykenischen Gesellschaftsstruktur. Als Dokumente mykenischen Palastmanagements beschäftigen sich die Linear B-Texte nicht mit Angelegenheiten, die außerhalb des Palastinteresses lagen. So bieten sie ein sehr einseitiges Bild mykenischen Lebens einschließlich der Sozialverhältnisse. Chronologisch auf ein Jahr beschränkt ${ }^{3}$, enthalten sie keine Daten für eine diachrone Untersuchung der historischen Entwicklung(en) der mykenischen Sozialstruktur und Sozialbedingungen. Sie sind eine Momentaufnahme ${ }^{4}$ der mykenischen Gesellschaft(en),

1 Derzeit c.4.500 Linear B-Texte auf Tontäfelchen aus Knossos, Pylos, Theben, Mykene und Tiryns, sowie aus diversen mykenischen Fundorten eine Reihe von Vasen mit aufgemalten kurzen Linear B-Inschriften. Für Literatur s. Fn. 2. - Manche Fakten, die in dem vorliegenden Text erwähnt werden, mögen für Mykenologen elementar und allbekannt erscheinen; bei dem vorwiegend aus Orientalisten bestehenden Teilnehmer- und Interessentenkreis des Symposions von Haifa dagegen konnte ihre Kenntnis nicht als selbstverständlich vorausgesetzt werden.

2 Zu den physischen Eigenschaften der Linear B-Tafeln und zum Inhalt der Texte siehe M. Ventris-J. Chadwick, Documents in Mycenaean Greek (2. Aufl. von J. Chadwick), Cambridge 1973; J. CHADwick, The Mycenaean World, Cambridge 1976, bes. S. 15-33; Informationsstand und Bibliographie zuletzt zusammengefaßt in A. MORPURGo DAviesY. Duhoux (Hg.), Linear B: A 1984 Survey, Louvain 1985, bes. der Beitrag von Y. Duнoux, S. 7-74 zu den Täfelchen selbst und zur Linear B-Schrift.

${ }^{3}$ Nicht für längeren Gebrauch vorgesehen (s. J. T. KILLEN, op. cit. [Fn. 8], S. 243), wurden die Täfelchen lediglich an der Luft getrocknet, aber nicht im Brennofen gehärtet. Die erhaltenen Texte verdanken wir paradoxer Weise der Härtung der Täfelchen im Katastrophenbrand der Paläste. Daher beziehen sie sich nur auf jenes Verwaltungsjahr, in dem der jeweilige Palast niederbrannte (s. ferner Fn. 4 und 5).

4 S. vorige Fn. - Genau genommen, betreffien die Texte nicht einmal das ganze jeweilige Verwaltungsjahr, sondern nur die Zeit bis zur Zerstörung des Palastes, mitunter 
zumeist aus der Zeit des Unterganges der mykenischen Paläste und des Endes der mykenischen Palastperiode ${ }^{5}$. Die Erforschung der mykenischen Sozialstruktur ist weiter behindert durch die Beschränkung der Texte ausschließlich auf die Belange der Palastwirtschaft. Dadurch müssen wir uns mit zufälligen und oft zusammenhangslosen Informationsstücken zur Gesellschaft zufriedengeben, die hinter dieser Wirtschaft stand. Oft sind es nur Listen mit Namen, Titeln und Berufstermini, von denen viele bis heute ohne eine zufriedenstellende Deutung geblieben sind $^{6}$. Das Fortleben eines Teiles der mykenischen Sozialterminologie in das klassische Griechisch macht die Sache nicht leichter, da in jedem Fall zu fragen ist, wie weit mit einem Terminus auch seine Bedeutung den Untergang der mykenischen Paläste und Staaten überlebt haben mochte?

Angesichts des Fehlens mykenischer Texte, die Literatur, politische und historische Fakten, Korrespondenz, Zeugnisse der Religiosität etc. enthalten, bleibt nichts anderes übrig, als von diesen problematischen Linear B-Tafeln aus und aus dem Blickwinkel der mykenischen Palast-

also nur wenige Monate. Was während dieser Zeit nicht notiert wurde, bleibt uns unbekannt.

s Auf dem griechischen Festland markieren die Zerstörungsschichten der Paläste, in denen man Linear B-Texte fand, mit wenigen Ausnahmen das Ende der archäologischen Stilperiode Späthelladisch (= SH) III B (Ende 13. Jh. v. Chr.) und zugleich das Ende der mykenischen Palastzeit überhaupt: es folgte kein Wiederaufbau der Paläste nach dem Ende von SH III B. Somit bezieht sich der Inhalt der Texte in den meisten Fällen auf das allerletzte Jahr (s. Fn. 3 und 4) nicht nur des betreffenden Palastes, sondern der mykenischen Palastzeit insgesamt. - Die ersten mykenischen Paläste in dem auf S. 33 definierten Sinn entstanden nach heutigem Wissensstand in der archäologischen Periode SH II B/ IIl A 1. Somit dauerte die mykenische Palastzeit vom späteren 15. Jh. bis zum Ende des 13. Jh. v. Chr. - Die Datierung der Knossos-Texte wird heftig debattiert. Entweder datieren sie gleichzeitig mit den älteren mykenischen Palästen des Festlandes, oder sie gehören ebenso an das Ende des 13. Jh. wie der Großteil der festländischen Texte. Ich persönlich bin eine Anhängerin der höheren Chronologie.

- Die Linear B-Zeichen, die für offiene Silben stehen, eignen sich nur schlecht für die Wiedergabe des Griechischen. Nicht alle Silbenfolgen (= Wörter) sind daher selbstevident, vor allem dann, wenn diese Wörter nicht ins Lexikon des 1. Jahrtausend v. Chr. übernommen wurden. Besonders problematisch sind hapax legomena, aber auch termini technici und Personalbezeichnungen, vor allem dann, wenn sie mit den Palästen untergegangen sind. Bei manchen besteht der Verdacht, daß sie nicht einmal griechisch bzw. indogermanisch sind. Für Literatur s. Fn. 2, zur Problematik der Interpretation von Namen, Berufsbezeichnungen und Titeln M. LindGren, in Colloquium Mycenaeum (s. Fn. 7), S. 81-86.

$7 \mathrm{Vgl}$. etwa die konträren Standpunkte und Ergebnisse von A. Morpurgo Da vies und F. GsChNitzer zu diesem Thema, beide in E. Risch und H. Mühlestein (Hg.), Colloquium Mycenaeum, Neuchätel 1979, S. 87-108, bzw. S. 109-134. 
ökonomie zu versuchen, einen Einblick in die sozialen Verhältnisse Griechenlands während der mykenischen Zeit zu gewinnen.

Es wurde oft behauptet, daß das mykenische Wirtschaftssystem, wie es sich in den Linear B-Texten spiegelt, ähnlich wie die gleichzeitigen Wirtschaftssysteme der Staaten des Alten Orients organisiert und strukturiert war. Kürzlich hat ein "1984 survey« der Linear B-Studien diese Ansicht kraftvoll und überzeugend untermauert ${ }^{8}$. Ihr Autor hat darüber hinaus gezeigt, daß diese Form der Wirtschaft nicht getrennt werden kann von der überragenden, zentralen und multifunktionalen Rolle eines Palastes, wie sie den Teilnehmern dieses Symposions wohlbekannt ist. Wie ihre orientalischen Gegenstücke, waren mykenische Paläste nicht nur Residenzen und Hofhaltungen der Monarchen. Sie beherrschten vielmehr, mit Hilfe einer zentralen Bürokratie, praktisch alle Aspekte des öffentlichen Lebens, also Politik, Kultur, Wirtschaft, Religion, Rechtssprechung und Heerwesen und alles weitere. Wenn man ein solches System als "Near Eastern economy" oder "Asiatic economy ${ }^{9}$ bezeichnet, erweitert man den Begriff "Wirtschaft" zu einem der "Staatsform", und der marxistische Begriff "asiatische Despotie" trifft den Sachver halt auch nicht ganz. Ich würde es vorziehen, von einer "Palastherrschaft" oder einem »Palaststaat" zu sprechen, auch in Fällen, wo dieses System ein Großreich umfaßte. Im Alten Orient war diese Staatsform typisch für die 2. Hälfte des 2. Jahrtausends v. Chr. und war eng verbunden mit der Herausbildung oder Umorganisation von Staaten und Reichen mit einer starken zentralen monarchischen Gewalt, die die absolute Herrschaft beanspruchte und mittels einer zentralen und komplizierten Bürokratie ausübte. Archäologische Quellen ${ }^{10}$ und die Aussagen der Linear B-Texte zeigen, da $\beta$ ein ähnlicher Proze $B$ in zumindest einigen Staaten der mykenischen Welt während der selben Periode stattgefunden hat.

8 J. T. KILLEN, The Linear B Tablets and the Mycenaean Economy, in Linear B: A 1984 Survey (s. Fn. 2), S. 241-305.

9 J.T. KILlEN, op. cit., folgt der Terminologie von M. I. FinLEY, formuliert etwa in dessen The Ancient Economy, London 1973, S. 28 f.

10 Zur Präponderanz der Paläste in Siedlungsstruktur und Architektur der myk. Staaten Messeniens und der Argolis K. Kilian, in Praktika tou defterou synedriou Messeniakon spoudon (Kyparissia 1982), Athen 1984, S. 55-68. - Zur Ausbreitung des Herrschaftsgebietes von Pylos auf Kosten kleinerer Staaten der frühmykenischen Zeit S. Deger-Jalkotzy, E-QE-TA. Zur Rolle des Gefolgschaftswesens in der Sozialstruktur mykenischer Reiche, Wien 1978, S. 201. - Zur Zentralisierung der industriellen Produktion und der Warenlagerung im Palast von Pylos J. C. Wright, in C. Shelmerdine-Th. Palaima (Hg.), Pylos Comes Alive, New York 1984, S. 19-29. 
Unter diesen Voraussetzungen mag es nicht überraschen, wenn einige Aspekte des mykenischen Sozialsystems ebenfalls Ähnlichkeiten mit den Verhältnissen in den gleichzeitigen Staaten des Alten Orients aufweisen. Wie die Beiträge von Herrn Hiller und Herrn Uchitel zu diesem Symposion zeigen, trifft dies vor allem auf jene Institutionen und Strukturen zu, die den Erfordernissen des Palaststaates und seiner Verwaltung entsprechen. Doch die Mykenäer adoptierten nicht einfach ihre Vorbilder ${ }^{11}$, sondern adaptierten sowohl die architektonische Ausgestaltung ${ }^{12}$ wie auch die Organisation und Verwaltung der Paläste ${ }^{13}$ entsprechend ihren eigenen Traditionen und Verhältnissen und ihrer eigenen Umwelt ${ }^{14}$. Wenn man die mykenischen Institutionen mit Hilfe eines Vergleiches mit jenen des Alten Orients verstehen will, muß man das Prinzip von mutatis mutandis stark berücksichtigen.

Meiner Meinung nach haben die Mykenäer ihre Fähigkeit zur kreativen Adaptation auch auf einem anderen Sektor der Verwaltung bewiesen, wo ebenfalls Wirtschaft und Sozialstruktur eng miteinander verbunden sind, nämlich bei Landverteilung und Landbesitz ${ }^{15}$.

Hauptquelle dafür sind die Texte von Pylos in Messenien ${ }^{16}$. Dort

1 Vielfach ist man sich heute darüber einig, daß die mykenische Kultur in allen ihren Aspekten nicht allein dem anregenden Vorbild des minoischen Kreta verpflichtet war. Auch die eigenen, helladischen Traditionen wirkten prägend. Das früher übliche Asyndeton "minoisch-mykenisch" sollte für die beiden großen ägäischen Kulturen des 2. Jahrtausend v. Chr. nicht weiter verwendet werden. - Zum Verhältnis der mykenischen Kultur zu orientalischen Vorbildern und Einflüssen s. die folgenden Fn.

12 Unterschiede zwischen der minoischen und der mykenischen Palastarchitektur betonte zuletzt D. Preziosı, Minoan Architectural Design, Berlin-New York-Amsterdam 1983. Ebenso K. KiliaN, in Archäologisches Korrespondenzblatt 14 (1984), S. 37-48; K. Kilian wies ferner beim Straßburger Kolloquium auf die helladischen Traditionen in der mykenischen Palastarchitektur hin (im Druck). - Weiter s. auch Fn. 14 und 28.

13 Vor allem J. T. KILleN, op. cit. (Fn. 8). Offien bleibt, ob sich die mykenischen Palaststaaten im 15. Jh. v. Chr. aufgrund direkter orientalischer Vorbilder, oder über Vermittlung durch das minoische Kreta herausbildten und organisierten. - S. ferner unten S. 48 und Fn. 88.

14 S. bereits Fn. 11 und 12. - Nach K. KILIAN, Zur Funktion der mykenischen Residenzen auf dem griechischen Fesıland (im Druck für die Akten d. Kolloqu. d. Schwed. Schule in Athen 1984) weisen einzelne Elemente in der Anlage mykenischer Paläste funktionale Parallelen zu orientalischen Palastanlagen auf, bleiben aber ohne Entsprechung in der architektonischen Gestaltung (s. weiter auch Fn. 28). - Zur Übernahme des Streitwagens aus dem Orient und zur Herausbildung der entsprechenden Sozialorganisation in frühmykenischer Zeit S. Deger-JALkotzy, op. cit. (Fn. 15), bes. S. 108-111.

15 Dazu bereits S. Deger-JALkotzy, Zum Charakter und zur Herausbildung der mykenischen Sozialstruktur, in A. Heubeck-G. NeumanN (Hg.), Res Mycenaeae, Göttingen 1983, S. 89-111.

${ }_{16} \mathrm{Zu}$ den E-Serien von Pylos grundlegend M. Ventris-J. Chadwick, Documents, 2. Aufl. (s. Fn. 2). Auffallend die getrennte Buchführung von einerseits /dämos/-Land in den $\mathrm{Eb} / \mathrm{Ep}$-Texten, andererseits von ki-ti-me-na-Grundstücken in den En/Eo-Texten. Dabei 
findet man unter den diversen Begriffen für Landbesitz und Landeigen-

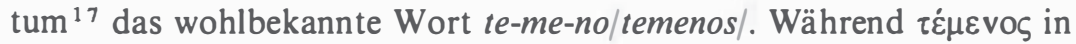
klassischer Zeit im allgemeinen die Bedeutung von Tempelbezirk bzw. Landbesitz einer Gottheit hatte, bezeichnete es in mykenischer Zeit Land, das dem König - wa-na-ka/wanaks/äva $\xi$ - gehörte. Eine genaue Definition ist allerdings durch die spärliche Bezeugung des Wortes ${ }^{18}$ nicht möglich. Ausgehend vom Gebrauch des Wortes $\tau \dot{\varepsilon} \mu \varepsilon v o \varsigma$ in klassi-

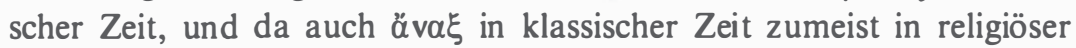
Bedeutung (als Anrede für Götter) verwendet wurde ${ }^{19}$, wollte man verschiedentlich wa-na-ka wie auch te-me-no für die mykenische Zeit der sakralen Sphäre zuweisen. Das ist aber m.E. nicht nötig. Abgesehen davon, daß die alten Kulturen keine so säuberliche Trennlinie zwischen dem Sakralen und dem Profanen zogen, wie wir es gewöhnt sind, zeigt

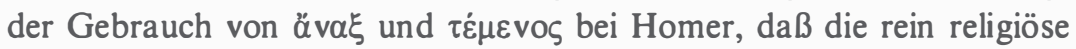
Begrifflichkeit der beiden Termini nicht die ursprüngliche war ${ }^{20}$. Ilias und Odyssee verwenden a̋va $\alpha$ als Anrede für Könige, und $\tau \dot{\varepsilon} \mu \varepsilon v o \varsigma$ dient als Bezeichnung für ein Stück Land, das vom übrigen Grund und Boden abgesondert wurde ${ }^{21}$ — etymologisch gehört das Wort ja $z u \tau \varepsilon \dot{u} \boldsymbol{v} \omega$ "zerschneiden, abschneiden, abtrennen" - und von der Gemeinde dem König zur Verfügung gestellt wurde, solange er an der Macht war ${ }^{22}$. Belege auch bei anderen Völkern für diese Idee der "Königsflur « ${ }^{23}$ legen es nahe, hier die ursprüngliche Bedeutung des Begriffes zu sehen, auch wenn im Mykenischen sakrale Aspekte des Königtums dazugekommen sein mochten. Das mykenische/temenos/ war das Landeigentum des Königs, das aus dem übrigen Landverteilungsschema ausgenommen war.

Es ist nicht nötig, nach dem Alten Orient für eine Erklärung von /temenos/ zu schauen. Die innergriechische Parallele des Homerischen

enthalten die Eb- bzw. die Eo-Texte jeweils die Erstaufnahme von Einzeldaten, die Epbzw. die En-Serien dagegen für jeden Sektor eine geschlossene Gesamtdarstellung durch einen Hauptschreiber. - Mit Landverteilung hängen weiters PY Un 718, und wohl auch die Texte der Na-Serie zusammen (J. CHADwick, in Documents, 2. Auf., S. 469). Zusammenfassend, mit Bibliographie, zuletzt J. T. KILLEN, op. cit. (Fn. 8), S. 243-250.

17 Terminologische Differenzierung bei S. Deger-Jalkotzy, op. cit. (Fn. 15), S. 100.

18 Nur PY Er 312.1,3.

19 Nach Isokrates, Euag. 72 Anrede für Prinzen und Prinzessinnen auf Zypern im 4. Jh. v. Chr.

20 S. Deger(-Jalkotzy), Herrschafisformen bei Homer, Wien 1970, S. 47-55.

${ }^{21}$ II. VI 192-95; IX 577-80, vor allem XVIII 550-58. Od. VI 293; XI 185 et al.

22 S. Deger, op. cit. (Fn. 20), Anm. 415. Jetzt auch M. I. FinLeY, The World of Odysseus, 2. Aufl., London 1977, S. 95 (anders in Historia 6 [1957], S. 148 ff.).

${ }^{23}$ S. DEGER, op. cit. (Fn. 20), S. 104 f. 
$\tau \varepsilon \dot{\mu} \mu \varepsilon v o \zeta$ und die erwähnten außergriechischen Beispiele von "Königsland " bei anderen, namentlich indogermanischen Völkern ${ }^{23}$, schließlich aber die recht naheliegende allgemeine Idee eines königlichen Privateigentums lassen das /temenos/ der Linear B-Texte im Sinne des königlichen Grundeigentums als eigenständige mykenische Institution erscheinen, wie immer auch ihre engere Charakteristik und ihre historische Entwicklung gewesen sein mochten.

Bemerkenswert ist aber, daß neben dem /wanaks/ noch eine zweite Person ein /temenos/ besaß, ein Mann mit dem Titel ra-wa-ke-ta/lāwāgetās/. Sein /temenos/ betrug nur ein Drittel des Flächenausmaße des königlichen/temenos/, was zu der communis opinio geführt hat, daß es sich hier um den zweiten Mann im Staat von Pylos handelte. Über seine genaue Position ist nichts zu eruieren ${ }^{24}$. Der Titel - eine Zusam-

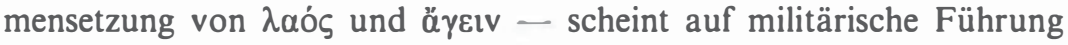
hinzuweisen, doch kann sich die Anwendung von Titeln bekanntlich weit von ihrer ursprünglichen Bedeutung entfernen. Den Texten nach dürften die Aktivitäten des /läwägetās/ tatsächlich auch militärische Funktionen umfaßt haben ${ }^{25}$, scheinen aber im übrigen denen des /wanaks/ in mancherlei Hinsicht ähnlich gewesen zu sein. Der Besitz des /temenos/ deutet ebenfalls auf eine dem König vergleichbare Stellung, wenn nicht überhaupt auf eine Teilhabe am Königtum. Besonders interessant ist aber der Umstand, daß ihm eine eigene Abteilung von palastabhängigem Personal unterstand, das als ra-wa-ke-si-jo /lāwägesios, -oi/ bezeichnet wurde ${ }^{26}$. Eine Funktionsanalyse der archäologischen Zeugnisse mykenischer Paläste hat kürzlich die Existenz eines Sekundärpalastes neben dem großen Megaron-Zentralkomplex als charakteristische Eigenart mykenischer Paläste aufgezeigt ${ }^{27}$. Ich halte es für sehr wahrscheinlich, daß /wanaks/ und /läwägetās/ mit dieser spezifischen Form eines Doppelpalastes (Haupt- und Nebenpalast) in Beziehung zu setzen sind, obschon Charakter, Zweck und historischer Hintergrund dieser Doppelung aus den Texten nicht erklärt werden können ${ }^{28}$.

24 Zusammenstellung der ra-wa-ke-ta-Daten bei M. Lindgren, The People of Pylos, Uppsala 1973, Bd. II, S. 134-136.

2s Zuletzt J. Chadwick, The Muster of the Pylian Fleet (im Druck für Akten des 8. Mykenolog. Kolloquiums Ochrid 1985).

26 Z.B. su-qo-ta "Schweinehirt", "a-mo-te-u "Wagner«, qo-u-ko-ro "Rinderhirt", u.ä. Vgl. S. Deger-Jalkotzy, Noch einmal zur Ea-Serie von Pylos (im Druck).

27 K. Kilian, s. Fn. 14.

28 K. Kilian, op. cit. (Fn. 14) konnte für den mykenischen Doppelpalast keine ägäischen Vorbilder finden, wohl aber im syro-palästinensischen Raum zumindest eine gewissen Parallele in der Existenz zweier zeitgleicher Palastanlagen in derselben Siedlung. 
Abgesehen von/temenos/, scheint das Grundeigentum im mykenischen Staat von Pylos nach einem doppelten System aufgeteilt gewesen zu sein, das offenbar eng mit der Sozialstruktur des Staates verbunden war. In dem einen System dominiert der Begriff ko-to-na ki-ti-me-na, im anderen die Kategorien ke-ke-me-na ko-to-na und ka-ma.

Zwei Termini kommen in beiden Sektoren vor, ko-to-na und o-na-to. Ko-to-na $\mid k t o i n a \bar{a} /$ ist verwandt mit $\kappa \tau i \zeta(\omega)$ im Sinne entweder von "besiedeln, ansiedeln, kolonisieren«, oder von »bebauen, kultivieren«. Die Originalbedeutung ist schwer zu bestimmen, zumal auch ki-ti-me-na zur Wortfamilie $\kappa \tau i \zeta \omega$ gehört ${ }^{29}$. Zur Zeit der Abfassung der Pylos-Texte jedenfalls hieß $|k t o i n \bar{a}|$ soviel wie "Grundstück, Parzelle«. - O-na-to

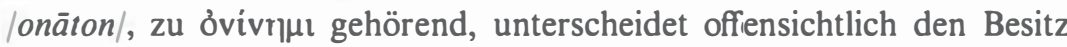
oder das Eigentum eines Grundstückes von der Nutznießung. Es bedeutet so etwas wie »Pacht«, »Fruchtgenuß«, "Nutznieß«.

Wenn wir uns zuerst dem Sektor von ka-ma und ke-ke-me-na ko-to$n a$ zuwenden, so ist der Unterschied zwischen diesen beiden Begriffen nicht evident. Eine häufige Phrase lautet: „N.N. hat die Pacht (den Fruchtgenuß od.dgl.) eines Grundstückes vom kek.-Typ beim (vom) |dāmos| ...u. |Dāmos/ist ein weiterer wohlbekannter Terminus. Wie das klassische $\delta \tilde{\eta} \mu \sigma_{\text {; }}$ mußte er eine Siedlungsgemeinschaft bezeichnet haben und wurde anscheinend für die ländlichen Siedlungsgemeinden (der mykenischen Siedlungsstruktur nach wohl Dorfgemeinden) außerhalb des Palast- und Stadtterritoriums verwendet. Die uns erhaltenen Texte beziehen sich auf pa-ki-ja-ne, eine in der Nähe von Pylos selbst gelegene Gemeinde, die eine prominente Rolle im Kult spielte. Dort nun lag ein beträchtlicher Teil des ke-ke-me-na-Landes beim /dämos/, da von diesen Grundstücken keine /onäta/ aus der Hand von Einzelpersonen, sondern nur vom /dāmos/vergeben wurden. Aber auch in den Texten der sich auf einen anderen Ort beziehenden Ea-Serie von Pylos kommt eine Vergabe von /onāta/ im ke-ke-me-na-Bereich durch Einzelpersonen nur selten vor.

Umgekehrt werden |onāta/ von ka-ma-Grundstücken von Einzelpersonen vergeben, nicht vom $|d a \bar{m} o s|^{30}$. Dies ist ein Unterschied zwi-

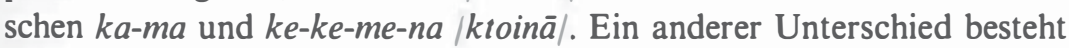

Ob diese funktionale Parallele auch soziologisch für die mykenische Herrschaftsstruktur und insbesondere für die Rolle des /läwägetās/ relevant ist, kann nicht ohne eingehende Untersuchung entschieden werden.

29 Siehe ferner S. Deger-Jalkotzy, op. cit. (Fn. 15), S. 96 mit Anm. 38, S. 109.

${ }^{30}$ Standardformel: N.P. (Nom.) e-ke ka-ma o-na-to pa-ro N.P. (Dat.) ka-ma-e-we ... "N.N. hat das jonāton/ einer ka-ma beim (vom) ka-ma-e-u namens N.N....". 
darin, daß ka-ma stets mit dem Verbum wo-ze-e /wordzehen/, einer

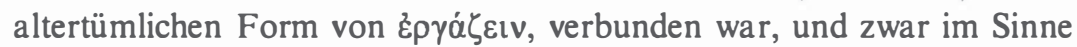
einer Verpflichtung: die Phrase lautet o-pe-ro wo-ze-e lophēlōn wordzehen/ "verpflichtet zu /w./«. Dagegen ist ke-ke-me-na-Land von dieser Obligation nur dann betroffen, wenn es sich um mindestens 2 /ktoinai/ handelte, oder wenn der Inhaber den Status eines ka-ma-e-u hatte ${ }^{31}$. Texte wie PY Ed 236 und PY Ep 613.3 zeigen, daß ka-ma-e-u nicht einfach jemand war, der ka-ma hatte. Vielmehr war ka-ma-e-u jemand, der zu /wordzehen/ verpflichtet war. Dazu paßt, daß es zu diesem Verbum kein nomen agentis gibt ${ }^{32}$.

Es ist anzunehmen, daß die beiden erwähnten Dinge nicht allein den Unterschied zwischen ka-ma und ke-ke-me-na /ktoinä/ a usmachten, doch ergibt sich aus den Texten nichts Näheres ${ }^{33}$. Die in einigen Tafeln der Ed-Serie von Pylos ${ }^{34}$ enthaltene Bilanz zeigt jedenfalls, daß beide Typen administrativ derselben Kategorie zugeordnet waren, wobei es offenbar weniger auf die genaue Zugehörigkeit zu einem der beiden Typen ankam als auf die Erfüllung der/wordzehen/-Obligation. Wenn wir nun schon eingangs festgestellt haben, da $\$$ die Linear B-Texte nur Tatsachen festhalten, die für den Palast wichtig waren, so folgt daraus, da $B$ es der Palast war, dem die Obligation des/wordzehen/entrichtet werden mußte.

$\mathrm{Da}$ ka-ma und ke-ke-me-na ko-to-na derselben Kategorie von Landinhabung zugehörten, ergibt sich auch daraus, daß sie gemeinsam in der Serie Ep von Pylos, also im selben Dokument verzeichnet sind. Die Verbindung von ke-ke-me-na ko-to-na mit der lokalen Siedlungsgemeinde, dem /dämos/, legt die Annahme nahe, daß auch ka-ma diesem Bereich zugehörte.

Im Unterschied zu ke-ke-me-na ko-to-na und ka-ma, war der andere Sektor des Grundbesitzes, jener von ko-to-na ki-ti-me-na, nicht mit dem /dämos/ verbunden, auch nicht mit irgendeiner anderen Körperschaft. Vielmehr befanden sich /ktoinai/ vom ki-ti-me-na-Typus in der Hand von Einzelpersonen, die als te-re-ta bezeichnet sind. Sie gaben /onäta/ von ihren Grundstücken an Leute weiter, die vielfach im Dienst des Palastes standen: religiöses Personal, abhängiges Palastpersonal sowie

31 Dazu S. Deger-Jalkotzy, E-QE-TA (Fn. 10), S. 68, und Res Mycenaeae (Fn. 15), S. 97 f.

32 Dagegen im ki-ti-me-na-Bereich das Verbum te-re-ja-e mit dem nomen agentis te-re-ta (siehe unten S. 45). Gegenüberstellung von ka-ma-e-we (nom. pl.) und te-re-ta /telestai/ in PY Ed 411 weist in dieselbe Richtung.

${ }^{33}$ Deger-Jalkotzy, op. cit. (Fn. 15), S. 97-99.

34 PY Ed 236, 317, 847, 901, Ed 411.2. 
Handwerker, die als /wanakteros/ angesprochen werden. In der Ea-Serie von Pylos gibt es auch Leute im Dienst des $/$ läwägetās $\left.\right|^{35}$, die im Besitz solcher Grundstücke waren.

Wenn also eine Zweigleisigkeit der beschriebenen beiden Landbesitzsektoren durch ihre getrennte Buchführung nahegelegt wird, so erscheinen sie dennoch auch miteinander verklammert, weil derselbe Personenkreis in beiden Sektoren auftritt. Besitzer von ki-ti-me-naParzellen finden sich als Inhaber auch von /dämos/-Land, und umgekehrt $^{36}$.

Die Situation war demnach kompliziert, und demgemäß sind die Versuche, die Landbesitzverhältnisse von Pylos zu verstehen, Legion. Weitgehend einig ist man sich dabei nur, daß ke-ke-me-na ko-to-na durch die Verbindung zu /dāmos/als Anteil an kommunalem Grundbesitz aufzufassen ist, und a uch ka-ma wird jetzt häufig diesem Bereich zugeordnet.

Davon ausgehend, wird ko-to-na ki-ti-me-na als gegensätzlicher bzw. komplementärer Begriff interpretiert, wobei man oft die Etymologien von ki-ti-me-na und ke-ke-me-na einbezieht. Ki-ti-me-na gehört als

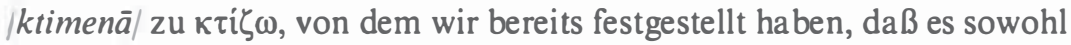
»besiedeln« als auch »bepflanzen, kultivieren« bedeuten kann, ähnlich dem deutschen Wort »bebauen«. Von den vielen für ke-ke-me-na vorgeschlagenen Interpretationen hat A. Heubeck nur zwei als linguistisch

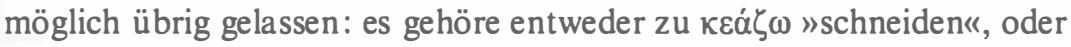

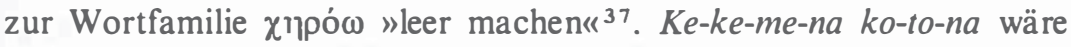
demnach entweder $/ k(h)$ ekhemenā ktoinā/ »leer gemachtes« oder »leer gelassenes (od. dgl.) Grundstück«, oder aber /kekesmenā ktoinā/38 ${ }^{38}$ "aufgeschnittenes, abgeschnittenes (od. dgl.) Grundstück».

Dem naheliegenden und häufig akzeptierten Gegensatzpaar "Brachland" (/kekhemenā ktoinā/) und "Pflanzland, kultiviertes Grundstück» ( kktoinā ktimenā/) hält J. Chadwick mit Recht entgegen, daß andere mykenische Termini, die ebenfalls $z u \kappa \tau i \zeta()$ gehören, eher für eine Bedeutung "besiedelt» von /ktimenā/ sprechen als für »bepflanzt« ${ }^{39}$. Auch von der intensiven Bodennutzung während der spätmykenischen Zeit her gesehen ${ }^{40}$, ist die Bedeutung "Brachland» nicht sehr wahr-

35 Dazu siehe oben.

36 Für weiteres siehe unten S. 46.

37 A. Heubeck, in Živa Antika 17 (1967), S. 17-21.

38 Diese Deutung hat den Nachteil, daß die bisher bekannten myk. Schreibregeln für /-sm-/ die Pleneschreibung *-se-me- verlangen würden.

39 Zuletzt op. cit. (Fn. 25). Vgl. auch Documents, 2. Aufl. (Fn. 2), S. 444.

40 H. KROLL, in Archäologischer Anzeiger, 1984, bes. S. $218 \mathrm{f}$. 
scheinlich; will man aber dennoch an /kekhemenä/ festhalten, wird man sich an eine andere Interpretation der Grundsemantik »leer machen, leer lassen « für ke-ke-me-na halten müssen ${ }^{41}$.

Ein anderes Gegensatzpaar, das /ktoinā ktimenā/ im Sinne von »Privatland " dem kommunalen/dāmos/-Land gegenüberstellt, ist in jüngerer Zeit mehr und mehr der Erkenntnis gewichen, daß der Palast kaum privates Land im engeren Sinn verzeichnet haben würde ${ }^{42}$. Dafür beschäftigt man sich mit der Frage, wie man sich angesichts der dominierenden Rolle des Palastes die Funktion des/dāmos/ innerhalb der Landbesitzordnung, aber auch allgemein im Rahmen der Wirtschaftsund Sozialstruktur der mykenischen Staaten vorstellen soll. Soweit ich sehe, gehen die Überlegungen in folgende Richtungen:

a) Alles verzeichnete Land gehört dem Palast, der /dāmos/ist nur eine lokale Verwaltungseinheit ${ }^{43}$.

b) Ki-ti-me-na- und ke-ke-me-na-Land sowie ka-ma sind /dämos/-Land verschiedener Kategorien ${ }^{44}$. Doch wird eingeräumt, daß der Palast wahrscheinlich der ultimative Eigentümer dieses Grund und Bodens war, jedenf alls aber einen weitreichenden Einfluß auf seine Verteilung und landwirtschaftliche Nutzung nahm ${ }^{45}$.

Mit solchen Überlegungen trägt man zwar dem Umstand Rechnung, $\mathrm{da} ß$ derselbe Personenkreis in beiden Sektoren aufscheint, und auch einander ergänzende Größenangaben der Bodenflächen werden als Argumente angeführt. Doch fällt es schwer zu verstehen, warum im |ktimenä/-Bereich die/wordzehen/-Obligation fehlt, und ebenso erhebt sich die Frage, warum die beiden Sektoren überhaupt verzeichnet wurden.

Noch in die Anfangsjahre der Linear B-Textinterpretation geht eine andere Auffassung zurück, die in den beiden Grundbesitzsektoren von Pylos zwei verschiedene Katgorien von Eigentum sieht. Während im $k a-m a$ - und ke-ke-me-na-Sektor die lokale Siedlungsgemeinde über das

41 Dazu unten S. 51-52.

42 K. WUNDSAM, Die politische und soziale Struktur in den mykenischen Residenzen nach den Linear B Texten, Wien 1968, S. 143, 165; Y. Duhoux, Aspects du vocabulaire économique mycénien, Amsterdam 1976, S. 63 f.; Zuletzt die Vorträge von P. DE Fidio (s. Fn. 92), J. Chadwick (s. Fn. 25), P. Carlier beim 8. Intern. Mykenolog. Kolloquium 1985 in Ochrid (im Druck).

${ }_{43}$ Vgl. etwa K. Wundsam, op. cit.; J. T. Killen, op. cit. (Fn. 8). Auch der Vortrag von Professor Hiller beim hiesigen Symposion scheint von dieser Voraussetzung auszugehen.

${ }^{44}$ P. De Fidio (s. Fn. 92) und P. Carlier (s. Fn. 42), mit unterschiedlicher Argumentation.

${ }^{45}$ P. De Fidio (s. Fn. 92). 
Land verfüge, sei ko-to-na ki-ti-me-na als Palasteigentum anzusehen ${ }^{46}$. Wie ich schon an anderer Stelle dargelegt habe, scheint mir dieser Auffassung viel Wahrscheinlichkeit zuzukommen. Als Argumente führte ich dabei Indizien aus der inneren Evidenz der Texte an und vor allem solche aus einem weit gespannten Vergleichsmaterial ${ }^{47}$ aus den zeitgleichen Palaststaaten des Alten Orients ${ }^{48}$.

Als Ausgangspunkt dient die Bindung des Besitzes von /dāmos/-Land an die Erfüllung von Leistungen an den Palast, die als /wordzehen/ bezeichnet werden. Ein Text, der schon unzählige Male behandelt worden ist, erhält unter einem solchen Blickwinkel einen weiteren Interpretationsaspekt:

PY Ep 704.5 f. "Die Priesterin Eritha hat (scil. so und so viel Saatgut $=$ Land) und erklärt feierlich, daß sie es als $e$-to-ni-jo für die (wegen der, im Namen der) Gottheit habe. Der /dāmos/ sagt aber, sie hätte o-na-ta von /ktoinai/ des ke-ke-me-na-Typs ${ }^{49}$.

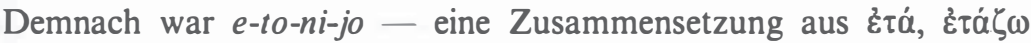
od. dgl. und óviv $\mu$ ı - eine vorteilhaftere Landinhabung als das übliche /onāton/. In einem anderen Zusammenhang wird berichtet, daß auch Gefolgsleuten des Königs, den sog. /hequetai/ ${ }^{50}$, diese vorteilhafte Nutzung ihrer Grundstücke, gelegentlich zugestanden wurde, und zwar sowohl im Fall von ka-ma-Grundstücken als auch von ke-ke-me-na ko-to-na. Bedenkt man die Bindung von ka-ma und (unter bestimmten Voraussetzungen, s. oben) von ke-ke-me-na-Parzellen an die Obligation /wordzehen/, so bietet sich als Interpretation des Begriffes e-to-ni-jo zwanglos die Befreiung von dieser Obligation an ${ }^{51}$. Die Priesterin Eritha verlangte also ein Privileg in der Form der Befreiung von der /word-

40 Vor allem L. R. Palmer, etwa in Transactions of the Philological Society, 1954, S. 24-45; ID., Achaeans and Indo-Europeans, Oxford 1955, S. 6; ID., Interpretation of Mycenaean Greek Texts, Oxford 1963, S. 191 f.

47 Im Unterschied zu Palmer, der allein das Hethitische Gesetzeswerk als Parallele heranzog, sonst aber mit indogermanischen Etyma und semantischen Parallelen operierte, s. auch unten S. 44 f. und Fn. 64.

48 S. Deger-Jalkotzy, op. cit. (Fn. 15). Siehe unten S. 45.

49 Der Paralleltext Eb 297 (zur Eb-Serie s. Fn. 16) bringt anstelle von da-mo den Begriff ko-to-no-o-ko /ktoinohokhoi/, wörtlich »Inhaber von /ktoinai/«. Die Träger dieses Titels mußten ein mit der Landverteilung befaßtes Gremium innerhalb des/dāmos/gewesen sein, vgl. M. Lejeune, in RÉG 78 (1965), S. 9; M. LindGren, op. cit. (Fn. 24), Bd. II, S. 88 f.; S. DeGer-Jalkotzy, E-QE-TA (Fn. 10), S.66-69, mit Literatur.

so S. Deger-Jalkotzy, op. cit., bes. S. $198 \mathrm{f}$.

51 S. Deger-Jalkotzy, op. cit., S. 55, 69f. UUbernommen bei P. De Fidio, in Kadmos 22 (1983), S. 25 und J. CHADwick, Koll. Ochrid (Fn. 25). 
zehen/-Obligation, die mit ihren Grundstücken verbunden war, und begründete dies mit ihrer religiösen Funktion.

Interessant ist nun, daß der Palast in dem Streit zwischen dem /dāmos/ und der Priesterin um dieses Privileg keine Entscheidung traf. Das heißt doch wohl nur, daß der Palast nicht in die Grundbesitzverhältnisse des /dāmos/ eingriff, sondern daß diese Verteilung bei der Siedlungsgemeinde selbst lag. Wie wir schon früher konstatierten, war der Palast aber an der Erfüllung der /wordzehen/-Obligation(en) interessiert. Man muß daher annehmen, daß diese Obligationen dem/dāmos/ kollektiv auferlegt waren, und daß der/dāmos/zugleich mit der Aufteilung der Grundstücke auch die Anteile an der Kollektivbelastung der Gemeinde intern regelte ${ }^{52}$. Eine solche Vorstellung erhält eine Stütze auch dadurch, $\mathrm{da} ß$ in den Linear B-Texten Lieferungen von Abgaben häufig nach Ortsnamen und Ethnika registriert werden, ohne daß Aufzeichnungen über die Organisation dieser Abgabenlieferung vorliegen. Gleiches gilt für militärischen Dienst bei der Flotte und bei der Küstenwache. Auch hier scheinen die lokalen Siedlungsgemeinschaften die Aushebung und Entsendung ihrer Kontingente selbst organisiert zu haben, Allerdings übte der Palast durch Aufsichtsbeamte auf der lokalen Ebene ${ }^{53}$ seine Kontrolle über die Durchführung der geforderten Leistungen und Abgaben aus.

Wenn demnach der /dāmos/von pa-ki-ja-ne gegen die Ansprüche der Priesterin auftrat, dann wohl nicht nur aus Prinzip, sondern deshalb, weil ihre Befreiung von der/wordzehen/-Belastung den anderen Mitgliedern der Gemeinde geschadet hätte, da sie zusätzlich zu ihren eigenen Anteilen an der Kollektivbelastung auch jene der Priesterin hätten übernehmen müssen. In dieselbe Richtung weisen auch Texte wie PY Ep 613.6,7 oder PY Ep 539.5, die genau festhalten, wer im Fall eines /onāton/ die Obligation des /wordzehen/ auszuführen hat, der ka-ma-e-u, also der Besitzer des Grundstückes, selbst oder der /onātēr/, der Pächter. Unter diesem Gesichtspunkt kann man die Organisation des/dāmos/Sektors nach zwei Richtungen hin verstehen. Entweder überließ der Palast das betreffende Land dem /dämos/ zur eigenen Verwaltung und mischte sich in die innere Organisation nicht ein ${ }^{54}$. Oder das Land war von vornherein Eigentum des /dämos/, aber nicht als Individualeigentum

$52 \mathrm{Zu}$ den /ktoinohokhoi/ unsere Fn. 49.

53 Dazu unten, S. 46.

54 Der Unterschied dieser Deutung zur oben S. 40 unter a) zitierten Auffiassung mancher Autoren von der myk. Landverteilungsorganisation liegt in der Betonung der Autonomie des /dāmos/. 
der einzelnen Gemeindemitglieder, sondern als kollektives Eigentum der ganzen Siedlungsgemeinschaft. Die Belastungen, die den Gemeinden vom Staat $=$ Palast kollektiv auferlegt waren, sind entweder als Gegenleistungen für die Überlassung des Bodens aufzufassen, oder aber als Leistungen, die dem Palast in seiner Eigenschaft als staatlicher Herrschaftsträger in Anerkennung dieser Herrschaft zu erbringen waren ${ }^{55}$.

Zahlreiche Parallelen aus den zeitgleichen Staaten des Alten Orients belegen, daß eine solche Auffassung zumindest nicht unmöglich ist ${ }^{56}$. Hinzuweisen ist besonders auf die gut dokumentierten Verhältnisse in Ugarit, die Professor Heltzer eindrucksvoll dargestellt hat ${ }^{57}$, und die in vielen Fällen Anregungen dafür geben, in welcher Richtung man das dürftige mykenische Material interpretieren könnte. Jedenfalls gab es im gesamten Alten Orient das Phänomen einer Kategorie von Grundbesitz, die nicht Staatseigentum (= Eigentum des Palastes oder eines Tempels) war, sondern sich in Privathand befand. Allerdings handelte es sich nicht um Grundbesitz von Einzelpersonen, sondern um einen solchen von kommunalen Körperschaften, häufig von dörflichen Gemeinschaften. Den Teilnehmern dieses Symposions sind diese Dinge wohlbekannt, wie ihnen auch die Kontroverse geläufig ist darüber, ob dieser kommunale Grundbesitz als Privateigentum ${ }^{58}$ angesprochen werden kann oder nicht ${ }^{59}$. Jedenfalls war er mit Obligationen belegt, für die der universelle Ausdruck ilku gebraucht wird, und für deren Erbringung die Eigentumsgemeinschaft kollektiv verantwortlich war ${ }^{60}$.

Es liegt natürlich nahe, das mykenische/dāmos/-Land in Analogie zu solchem Kommunalgrundeigentum außerhalb des Palastlandes zu sehen. Ebenso ist man angeregt, den Terminus/wordzehen/ als mykenisches Äquivalent zu ilku aufzufassen, womit Leistungen wie Corvée, Abgaben, Militärdienst umrissen wären ${ }^{61}$. Eine Reihe von Linear B-Texten würde eine solche Interpretation durchaus ermutigen, doch würde ihre Behandlung in diesem Rahmen zu weit führen ${ }^{62}$.

s5 Diese Unterscheidung ist rein juridisch, entsprechend der Definition von "Eigentum" und "Besitz" (dazu oben S. 35 und Fn. 17). Faktisch ist das Ergebnis gleich. S. ferner S. Deger-Jalkotzy, op. cit. (Fn. 15), S. 100-102, 108-111.

s6 Material bei S. Deger-JalkotZy, op. cit. (Fn. 15), S. 91-95.

57 M. HeltZer, The Rural Community in Ancient Ugarit, Wiesbaden 1976.

58 Dazu siehe Anm. 55.

59 Siehe auch unten S. 46r.

60 In den Hethitischen Gesetzen $\$ 46-50$, bzw. XXXVI und XXXIX, lautet der entsprechende heth. Terminus luzzi. Dazu aus jüngerer Zeit F. IMPARATI, op. cit. (Fn. 81). ${ }_{61}$ P. DE Fidio, in SMEA 23 (1982), S. 83-121 über die Abgaben der /damoi/ und ihre Organisation.

${ }^{62}$ Hinweise bei S. Deger-Jalkotzy, op. cit. (Fn. 15), passim. Ausführlichere Zusammenstellung bei P. DE FiDIo, Kolloquium Ochrid 1985 (s. Fn. 92). 
Logischer Weise führt eine solche Sicht des/dāmos/-Sektors weiter zur Annahme, daß es sich bei den /ktoinai ktimenai/ um Land im Eigentum des Palastes handelte ${ }^{63}$. Nun wird zugegebener Maßen nirgends in den Texten der $\mid k$ timena $/$-Sektor ausdrücklich mit dem Palast in Verbindung gebracht. Es gibt lediglich ein paar Hinweise dafür, da $ß$ es sich doch um Palastland gehandelt haben konnte. Zunächst ist daran zu erinnern, daß sich /ktoinai ktimenai/ in der Hand von Personen befanden, die den Titel te-re-ta trugen. Auf der Tafel PY Er 312 ist neben dem/temenos/ des /wanaks/ und jenem des /läwägetās/ auch der Grundbesitz von 3 als te-re-ta genannten Personen verzeichnet, und diese nächste Nähe zwischen te-re-ta und der monarchischen Spitze des Reiches wurde schon oft als Hinweis dafür angesehen, da $ß$ ihr Grundbesitz Palastland sein könnte. Ferner vertrat L. R. Palmer schon früh die Ansicht, daß der zu $\tau \dot{\varepsilon} \lambda$ os gehörende Titel te-re-ta/telestās/als "Dienstmann« bzw. "Lehensmann « zu verstehen sei, wenngleich die zur Argumentation von Palmer herangezogenen indogermanischen Bedeutungsparallelen problematisch waren ${ }^{64}$. Daß er mit seiner Interpretation doch das Richtige getroffen haben dürfte, habe ich anhand der innergriechischen semantischen Entwicklung von $\tau \dot{\varepsilon} \lambda \circ \varsigma$, wie er in den diversen Bedeutungen des Wortes im Griechischen des 1. Jahrt. v.Chr. faßbar wird, zu zeigen versucht ${ }^{65}$.

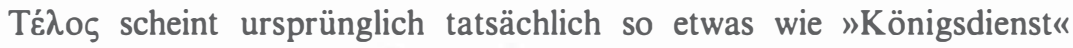
bedeutet zu haben, und /telestäs/ mochte der "Mann des königlichen Dienstes" gewesen sein. Ferner hat J. Chadwick wahrscheinlich gemacht, daß |ktoinā ktimena $\bar{a}$ | "besiedeltes" Land bezeichnete, "estate", also ein Gut mit einem Gutshof darauf. Diese Güter waren besiedelt mit |ktitai/, die nach PY An 724 dem Palastsektor zugehörten ${ }^{66}$. Auch /phutēres/, ländliche Arbeiter, die mit den Fruchtbaumkulturen zu tun hatten, siedelten allem Anschein nach auf Palastgütern ${ }^{67}$. Solche Palastabhängige waren also den /telestai/ unterstellt, und ebenso gaben die /telestai/ vorwiegend an Leute des Palastdienstes bzw. Palastpersonal

${ }^{63}$ So schon L. R. Palmer, op. cit. (Fn. 46).

${ }^{64}$ Besonders in Transactions of the Philological Society, 1954, S. 39 f. In seinen späteren Arbeiten hat PALMER auf die gewagten etymologischen und semantischen Verknüpfungen seiner te-re-ta-Interpretation verzichtet.

${ }^{65} \mathrm{Da}$ die Akten des 1. Internat. Kongresses über Messenien von Athen 1980 noch immer nicht im Druck sind, habe ich meinen Beitrag über die te-re-ta in Pylos in den weiteren Rahmen eines Aufsatzes über Landbesitz und Dienstpflicht in mykenischer Zeit eingearbeitet, der in Die Sprache erscheinen soll.

${ }_{60}$ J. Chadwick, Documents, 2. Auf. (s. Fn. 2), S. 444; ID., op. cit. (Fn. 25); ID., in BICS 26 (1979), S. 130.

67 Zu den mykenischen Fruchtbaumkulturen und zu den iphutères/ zuletzt St. Hiller, in A. Heubeck und G. Neumann (Hg.), Res Mycenaeae (s. Fn. 15), S. 186-188, 197 f. 
/onāta/ ihrer Grundstücke aus ${ }^{68}$. Ein weiteres Indiz liegt in dem nur zweimal belegten Wort te-re-ja-e, das am besten als /teleiähen/ zu deuten ist, "das tun, was zum */telos/ (Königsdienst) gehört "69. Dieser Terminus technicus wäre dann im Sektor der /ktoinai ktimenai/ das Äquivalent zu /wordzehen/ im /dämos/-Sektor. Daß/teleiähen/ so selten in den Texten bezeugt ist, versteht man zwanglos unter dem Aspekt, daß die Erfüllung der Pflichten des Königsdiensten $\left({ }^{*} /\right.$ telos/) automatisch mit dem Status eines /telestās/ verbunden war und erwartet wurde. Bezeichnender Weise finden sich die beiden Erwähnungen von te-re-ja-e dort, wo eine ungewöhnliche Regelung vorlag ${ }^{70}$. Welche Leistungen und Pflichten einem/telestäs/ oblagen, geht aus den Texten nicht direkt hervor. Sicherlich aber gehörte dazu die in den Tafeln vermerkte Tatsache, daß die /telestai/ Nutzanteile ihrer Ländereien bzw. Güter Leuten des Palastes überließen und ihnen auf diese Weise - im Namen des Palastes - eine Einkommensbasis boten. Militärdienst, Bekleidung von Ämtern und Abgaben an die Zentrale liegen als weitere verpflichtungen nahe und lassen sich aus einigen Texten erschließen ${ }^{71}$. Sie spiegeln sich wohl auch in der semantischen Entwicklung von $\tau \dot{\varepsilon} \lambda{ }_{0} \varsigma$ im klassischen Sprachgebrauch wider ${ }^{72}$.

Natürlich kommt dem eben entworfenen Bild eines Dualismus im Bereich des Grundeigentums und der damit zusammenhängenden sozialen Organisation der Bevölkerung des Reiches von Pylos ${ }^{73}$ nicht mehr als der Rang einer Hypothese zu. Das gilt aber in ebendemselben $\mathrm{Ma} B$ für alle anderen Interpretationen. Die Analyse der Texte für sich stößt eben in jedem Fall an die Grenze, daß die Position der te-re-ta und damit die Eigentumshoheit des $\mid k t i m e n a \bar{a}$-Sektor nicht wirklich evident sind. Die hier vorgelegte Interpretation hat den Vorteil, sich nicht nur

68 Vgl. oben S. 38 f.

69 C. WAtkins, Indo-European Studies, Harvard 1972, S. 219 zur Wortbildung. Mit der Semantik wird sich S. DEGER-JALKotZy (Fn. 65) auseinandersetzen.

70 S. Deger-Jalkotzy, s. Fn. 65.

7 S. Deger-Jalkotzy, s. Fn. 65.

72 Té̀os hat im Sprachgebrauch des 1. Jahrt. v. Chr. nicht nur die Bedeutung "militärische Einheit, Truppe, Aufgebot", sondern auch "territoriale Einheit, Bezirk ", und daneben "Amt, Amtswürde, Magistrat", bzw. Amtsträger, Beamter«, vor allem aber "Steuer, Abgabe, Zoll«. Dazu ausführlich mein te-re-ta-Beitrag von Athen (s. Fn. 65).

73 Wiederum ist auf die im Vergleich zu den altorientalischen Quellen mehr als dürftige Evidenz der Linear B-Texte hinzuweisen. Sie lassen viele Fragen unbeantwortet, die für die Beurteilung der myk. Gesellschaftsordnung von entscheidender Wichtigkeit wären wie z.B. der Status der /ktitai/ und der phutēres/ (s. oben und Fn. 66, 67) - Freie? Unfreie? Hörige? - oder auch die interne Organisation des /dāmos/. Selbst über die meisten Angehörigen des Palastsektors wissen wir kaum mehr als ihre Namen und/oder Titel. Das Bild bleibt in jedem Fall umrißhaft und vage. 
auf Etymologien von ke-ke-me-na, ki-ti-me-na und te-re-ta, und auch nicht auf Berechnungen der Maß- und Zahlangaben, die ihrerseits ebenfalls wieder auf Hypothesen beruhen, stützen zu müssen, sondern sich dazu auf Analogien aus dem Bereich des Alten Orients berufen zu können.

In einem Punkt treffen sich jedoch fast alle Ansichten, die in letzter Zeit zu unserem Thema geäußert wurden: zur Zeit der Abfassung der erhaltenen Pylos-Texte war der/dämos/-Sektor stark dem Zugriff des Palastes unterworfen. So figurieren/telestai/ nicht nur unter den Inhabern von /dämos/-Land, sondern gehören sogar dem Kreis der /ktoinohokhoi/, also dem Gremium an, das im /dāmos/ über die Zuweisung von Parzellen entschied ${ }^{74}$. Palastabhängiges Personal und Kultpersonal hoher und niedriger Ränge erhielt /onāta/ nicht nur von den /ktoinai/ der/telestai/, sondern auch vom/dāmos/, wobei die /wordzehen/-Obligation oftmals entfiel ${ }^{75}$. Gefolgsleute des Königs bekamen Landanteile beim /dāmos/ überhaupt als $e-t o-n i-j o{ }^{76}$. Ferner gab es Beamte, die entweder als Aufsichtsorgane des Palastes die Durchführung der Gemeindeleistungen überwachten, oder als verantwortliche interne Organe der Gemeinde selbst die Erfüllung der mit dem Grundbesitz der Gemeinde verbundenen Lasten verbürgen mußten, jedenfalls aber die Verbindung zwischen Siedlungsgemeinden und Zentralverwaltung gewährleisteten ${ }^{77}$.

Sehr interessant ist nun, daß ein ganz ähnliches Phänomen für die Staaten des Alten Orients während der 2. Hälfte des 2.Jahrtausend v.Chr. beobachtet wird, wo ebenfalls zwar einerseits neben dem staatlichen Grundbesitzsektor ein kommunaler parallel einherging, andererseits aber dem Palastsektor ein großes Übergewicht in den Grundbesitzverhältnissen zukam. Dieser Umstand hat früher verschiedentlich zu der (der Feudaltheorie verpflichteten) Vorstellung geführt, daß der gesamte Grund und Boden Eigentum der Krone, und die Gemeinde lediglich Verwaltungseinheiten gewesen wären ${ }^{78}$. Zwar widerlegt die moderne

${ }^{74} \mathrm{Zu}$ den /kloinohokhoi/s. Fn. 52.

75 In den Texten formuliert mit o-u-wo-ze /ou wordzei/ ner (sie) leistet die /w./-Pflicht nicht «. Zur Befreiung flächenkleiner Grundstücke von dieser Obligation sowie zu e-lo-ni-jo s. S. Deger-Jalkotzy, E-QE-TA (s. Fn. 10), S. 65-71.

76 Siehe oben S. 41.

77 In erster Linie gilt dies für die sog. ko-re-te-re, wie auch für den in PY Jn 829 und Jo 438 angeführten Personenkreis.

${ }^{78}$ So beispielsweise A. F. RaINEY für Ugarit (zitiert aus dem Hebräischen bei M. HeltZer, op. cit. [Fn. 57], S. 103). Für die mittelassyrische Sozialstruktur siehe J. N. PostGATE, in BSOAS 34 (1971), S. 496-520. 
Forschungsarbeit eine solche Auffassung mehr und mehr, doch besteht eine Unsicherheit und sogar eine Kontroverse darüber, ob der private Grundbesitzsektor mit der Zeit komplett vom staatlichen Sektor aufgesogen wurde 79 , oder ob der Eigentumsdualismus grundsätzlich beibehalten blieb ${ }^{80}$. Es ist also nicht die grundsätzliche Tatsache von getrennten Grundbesitzsektoren, die zur Debatte steht, sondern der Grad, in welchem der staatliche Sektor in den kommunalen eingriff. Nun hat Frau Imparati überzeugend vor Augen geführt, wie im Hethitischen Großreich die ursprünglich getrennten Sektoren von Palastland und Gemeindeland samt den damit verbundenen Obligationen im Laufe der Zentralisierung der Staatsgewalt und der Bürokratisierung der Verwaltung immer mehr ineinander flossen und die Grenzen verwischt wurden $^{81}$. Es ist gut möglich, daß sich mehr oder weniger ähnliche Prozesse in fast allen Staaten des späteren 2. Jahrtausends vollzogen, und daß die Präponderanz des staatlichen Grundeigentumssektors mit der Entwicklung der Palaststaaten-Systeme zusammenhing.

Was die mykenischen Verhältnisse betrifft, so hat die Überschneidung von /dämos/- und /ktimenāa/-Sektor zu den oben S. 40 unter a) und b) kurz charakterisierten Interpretationen geführt, die in unterschiedlicher Weise die Idee eines gemeinsamen Grundeigentümers beider Sektoren vertreten. Einig ist man sich aber darin, daß die Linear B-Texte bereits das Ergebnis eines längeren historischen Prozesses spiegeln, in dessen Verlauf ursprüngliche Strukturen sich fortentwickelt und gegenseitig überlagert hätten. Dieser Ansicht bin auch ich, und ich habe meine Vorstellungen als "diachrone These" an anderer Stelle formuliert ${ }^{82}$.

Gewi $B$ ist es unmöglich, in diesen grundsätzlichen Fragen eine Entscheidung zu treffen. Doch solange keine Texte vorliegen, die Auskunft darüber geben könnten, was im Fall des Ablebens oder sonstigen Verschwindens eines Grundeigentümers mit seinem Grundbesitz geschah, oder auch solange wir keine Verkaufsdokumente besitzen (was freilich bei dem Charakter und dem Inhaltsradius der Linear B-Texte auch nicht zu erwarten ist), und solange nicht erwiesen ist, daß die /telestai/ tatsächlich allesamt Mitglieder des/dāmos/ oder gar /ktoinohokhoi $\left.\right|^{83}$ waren ${ }^{84}$, und solange keine wirklich befriedigende Erklärung für die

79 Bekanntlich wird dieser Standpunkt vor allem von I. J. GeLb verfochten.

80 Protagonist dieser gegenteiligen Auflassung ist M. I. Diakonoff.

${ }^{81}$ Siehe besonders F. Imparati, Aspects de l'organisation de l'État hittite dans les documents juridiques et administratifs, in JESHO 25 (1982), S. 225-267.

${ }^{82}$ Op. cit. (Fn. 15), S. 108-110.

${ }^{83}$ Dazu s. Fn. 49.

84 Argumentation bei S. Deger-Jalkotzy, op. cit. (Fn. 10), S. 66-68. 
parallele Terminologie und Registrierung im Bereich des Grundbesitzes und der damit verbundenen sozialen Ordnung gefunden wird, glaube ich weniger an die oben S. 40 angeführten Interpretationen. In Analogie an die Staaten des Alten Orients denke ich vielmehr an zwei ursprünglich getrennte Eigentumskategorien, die einerseits im staatlichen Grundeigentum (Königsland, Palastland ${ }^{85}$ ), andererseits im Grund und Boden von Eigentumsgemeinschaften, zumeist von Dörfern ${ }^{86}$, bestanden. Der "private» Sektor wurde eigenständig verwaltet, aber er war an die Erfüllung von obligaten Leistungen gebunden, die an den Souverän in Anerkennung seiner Herrschaft zu entrichten waren. Diese Organisation geht meiner Meinung nach noch in die frühe mykenische Zeit zurück und hing mit der Einführung des Streitwagens und der Herausbildung einer aristokratischen Oberschichte zusammen ${ }^{87}$. Vorbild dafür waren die Verhältnisse im Alten Orient, und zwar noch vor der Zeit der Großreiche ${ }^{88}$. Die weitere Entwicklung verlief dann analog zu diesen. Mit der Herausbildung der mykenischen Palaststaaten unter einer einheitlichen monarchischen Gewalt, die mit Hilfe der Bürokratie eine straffe Verwaltung und Herrschaft ausübte, kam es vom 14. Jh. v.Chr. an $\mathrm{zu}$ einem Übergewicht des staatlichen (= Palast) Sektors, dessen große Schar von Dienstpersonal, über das Herr Hiller berichtet, erhalten

85 Anders als im Alten Orient, aber auch als im klassischen Griechenland, spielten die mykenischen Heiligtümer keine eigenständige wirtschaftliche Rolle im Staat. Wohl verfügten die Heiligtümer mancher mykenischer Gottheiten, den Linear B-Texten nach, über ein gewisses $\mathrm{MaB}$ an Wirtschaftsgrundlagen und -gütern, darunter auch Landbesitz (die Daten sind bei St. Hiller in AfO, Beiheft 19 [1982], S. 94-104 zusammengetragen). Es geht aber m.E. zu weit, daraus mit L. R. PALMER (vgl. etwa in Armées et fiscalité dans le monde antique [Colloques nationaux du CNRS, Paris 1976], Paris 1977, S. 35-62) die Vorstellung einer eigenen mykenischen Tempelwirtschaft ableiten zu wollen. Vielmehr war, ähnlich wie im Hethitischen Reich, in den mykenischen Palaststaaten der König (= der Palast) zumindest für die offizielle Religion und Kultausübung zuständig. Dazu gehörte die Ausstattung der Heiligtümer und ihres Personals mit den Grundlagen für ihren Lebensunterhalt. In diesem Sinne gehören Werkstätten, Viehherden und Ländereien im Besitz der Heiligtümer ursächlich zum Palastsektor der mykenischen Wirtschaft. Es gibt ja auch keine eigenen Archive der Heiligtümer, sondern ihre wirtschaftlichen Belange wurden im Palastarchiv festgehalten.

${ }^{86}$ Zur möglichen Existenz anderer lokaler Sozialformen mit kollektivem Grundeigentum J. Chadwick, in Documents, 2. Aufl. (s. Fn. 2), S. 469.471; J. T. Killen, in BICS 26 (1979), S. 133 f.; ID, op. cit. (Fn. 8), S. 248 f.; S. Deger-Jalkotzy, in Res Mycenaeae (s. Fn. 15), S. 110.

87 S. DeGer-JalKotzy, op. cit., S. $108 \mathrm{f}$.

88 Ibid., Wo diese Vorbilder genau zu suchen sind, läßt sich aufgrund der Quellenlage nicht sagen. F. SCHACHERMEYR, Griechische Frühgeschichte, Wien 1984, S. 60 f. und S. 6775, denkt in erster Linie an die Hethiter und an Ägypten. Doch kann man andere Kontaktzonen ebensowenig ausschließen wie eine Vermittlung durch das minoische Kreta (siehe auch Fn. 13). 
werden mußte. $\mathrm{Zu}$ den entsprechenden $\mathrm{Maßnahmen}$ gehörte unter anderem auch, daß der Palast auf Kosten der lokalen Gemeinden immer mehr in den privaten Eigentumssektor übergriff, indem Leute des Palastdienstes mit Grundstücken des/dāmos/-Landes zur Sicherung ihres Lebensunterhaltes ausgestattet wurden. Da nur ein Teil davon die unter /wordzehen/ subsumierten Obligationen erfüllte oder erfüllen mußte ${ }^{89}$, verringerte sich für den /dämos/ nicht nur die Bodenfläche, über die er frei verfügen konnte, sondern die kollektiv auferlegten Lasten des /wordzehen/verteilte sich auf immer weniger/dāmos/-Mitglieder. Angesichts des großen Bedarfes der Paläste an Nahrungsmitteln, um das abhängige Personal zu ernähren bzw. zu bezahlen ${ }^{90}$, sowie nach Rohstoffen für die Werkstätten der Palastindustrie, aber auch angesichts der großen Arbeitsleistungen, die für den Bau von Zitadellen und anderer Großprojekte wie auch für dem Umbau der Paläste im späteren Verlauf des 13. Jh. v.Chr. aufgebracht werden mußten, kann man sich unschwer vorstellen, daß die Belastungen in Form von Corvee und Abgaben, die den /dämoi/ aufgebürdet wurden, ähnlich erdrückend gewesen sein mußten wie in Ugarit ${ }^{91}$.

Viel diskutiert wird die Frage, warum die Landverteilungstexte von Pylos auf pa-ki-ja-ne und andere Bezirke im Umkreis des Palastes konzentriert sind, während für andere Bezirke kaum diesbezügliche Aufzeichnungen vorliegen ${ }^{92}$. Die Erklärung, daß in pa-ki-ja-ne als dem religiösen Zentrum von Pylos ein spezielles, von den anderen Bezirken unterschiedenes System geherrscht habe, kann nicht ausreichen. Denn die Na-Serie von Pylos ${ }^{93}$ ebenso wie das gelegentliche Auftreten der Landbesitztermini der E-Serien auch für andere Distrikte des Reiches, vor allem aber ihre Bezeugung in den Texten von Knossos und Tiryns beweisen, daß das Landbesitzsystem von pa-ki-ja-ne sowohl mit den allgemeinen Verhältnissen von Pylos, als auch überhaupt mit dem mykenischen Standard im Einklang war. Die Sonderstellung von pa-kija-ne und der übrigen in der Nachbarschaft des Palastes gelegenen Bezirke lag wohl eher darin, daß dort mehr Leute des Palastes ihren Grundbesitz hatten als in den weiter entlegenen Gebieten. Die E-Serien

89 Siehe weiter oben und Fn. 75.

90 Siehe den Vortrag von Prof essor Hiller bei diesem Symposium.

91 M. HeltZer, op. cit. (Fn. 57), Kapitel II.

92 Zuletzt J. T. Killen, op. cit. (Fn. 8), S. 245-247, mit Literatur. Auch P. DE Fidio, Le rôle des communautés de village dans le royaume de Pylos, Vortrag beim 8 . Internat. Mykenologischen Kolloquium in Ochrid 1985 (im Druck).

${ }_{93}$ J. T. Killen, op. cit. (Fn. 8), S. 245. 
von Pylos hatten m.E. den administrativen Zweck, die Versorgung der Leute des Palastdienstes - in pa-ki-ja-ne vornehmlich Kultpersonal mit Grundbesitz teils vom palasteigenen Land, teils vom kommunalen Sektor zu verzeichnen, bzw. ob sie die Obligationen des/wordzehen/im kommunalen oder des /teleiähen/ im palasteigenen Sekt or ${ }^{94}$ zu entrichten hatten oder nicht ${ }^{95}$. Bezüglich der anderen Bezirke, wo die Situation wahrscheinlich nicht so komplex war ${ }^{96}$, registrierten die Listen der zentralen Verwaltung weniger die Verteilung von Grund und Boden als hauptsächlich Art und Höhe der erwarteten Leistungen und deren tatsächliche Erfüllung oder Nichterfüllung. Sie beziehen sich einerseits auf die Verpflichtungen und Leistungen der $/$ dāmoi $\left.\right|^{97}$, und andererseits auf jene der Leute der diversen Ränge des Palastdienstes ${ }^{98}$.

Zusammenfassend hat es also den Anschein, als ob im mykenischen Reich von Pylos nur ein Teil des Grund und Bodens im Eigentum des Palastes war. Das persönliche Grundeigentum des Königs und jenes des /lāwāgetās/ waren überhaupt als /temenos/ herausgenommen. Ob es sich dabei um zusammenhängenden Grundbesitz handelte, oder ob das /temenos/ des Königs und jenes des /lāwāgetās/ aufgeteilt waren auf mehrere, in verschiedenen Regionen gelegene Grundstücke, ist angesichts der spärlichen Dokumentation nicht zu sagen. Ebensowenig ist evident, in welcher Weise dieser Grundbesitz genützt und verwaltet wurde. Abgesehen von /temenos/, war das Grundeigentum in Pylos in zwei Sektoren gegliedert. Ein Teil gehörte dem Palast und diente, je nach geographischer Lage, für Plantagen und/oder Herdenbetrieb. Ferner verfügte der Palast in jedem Bezirk ${ }^{99}$ über Landgüter, /ktoinai ktimenai/.

94 Dazu S. Deger-Jalkotzy, siehe Fn. 65.

$95 \mathrm{Zu} o$-u-wo-ze und e-to-ni-jo oben Fn. 75; Befreiung von Obligationen drückt sich vermutlich auch in dem Terminus e-re-u-te-ro(ra) "frei« bzw. e-re-u-te-ro-se |eleutherōse/ wer hat befreit" aus (J. CHADwICK, Documents, 2. Aufl. [Fn. 2], S. 469). Nichtleistung von Abgaben steht hinter $o-u$-di-do-si und $o-u$-di-do-to ssie liefern nicht , bzw. "... wird nicht geliefert«.

$96 \mathrm{Vgl}$. die globalen Bodenflächenangaben in PY Eq 213 und auch in An 830.6.

97 Dazu zusammenfassend zuletzt P. DE Fidio (s. Fn. 92). S. auch J. T. KILlEN, op. cit. (Fn. 8), S. 270 f.

98 Zur Rangordnung im palatialen Sektor der Sozialstruktur von Pylos S. DEGERJALKoTZY, op. cil. (Fn. 15), S. 105 f.

99 Zur wahrscheinlichen Gültigkeit der in den E-Serien erkennbaren Organisation für das ganze Reich von Pylos siehe oben. Die Situation dürfte ähnlich wie in Ugarit gewesen sein, wo sich die landwirtschaftlichen Betriebe des Palastes ebenso wie die an die "Leute des Königs« (bns $m l k$ ) verliehenen Palastländereien über die Gemeinden des Landes hin verteilten, vgl. M. HeltZER, The Internal Organization of the Kingdom of Ugarit, Wiesbaden 1982. Für die Periode IV von Alalah̆ beobachtet H. KLENGEL, „daß sich die Palastwirtschaft weniger auf die Hauswirtschaft in der Residenz selbst konzentrierte, 
Verwaltet wurde dieses Eigentum von den Beamten des Palastes ${ }^{100}$. Die höheren Ränge des palatialen Gesellschaftssektors und wohl auch die Mitglieder der Königsfamilie ${ }^{101}$ hatten Anteil an den Palastgütern, sei es in Form von Herden ${ }^{102}$ oder in Form von Grundbesitz. Letzteres betraf besonders die /telestai/, die für diesen Grundbesitz die Leistungen des /teleiähen/ schuldeten ${ }^{103}$. Vor allem sicherten sie durch die Überlassung von /onāta/ ihrer /ktoinai ktimenai/ den Lebensunterhalt weiterer Palastbediensteter, in pa-ki-ja-ne von Angehörigen des Kultpersonals. Auf den Palastländereien lebten ferner Landarbeiter wie /ktitai/ und /phutēres/, die zu den unteren Rängen der Leute des Palastdienstes gezählt haben mußten ${ }^{104}$.

Der andere Sektor des Grundeigentums lag in der Hand der jeweiligen lokalen Siedlungsgemeinschaft, des /dāmos/. Er unterlag der kollektiven Verpflichtung zu Leistungen gegenüber dem Palast, deren Beteiligung durch die Grundbesitzer im/dämos/-Sektor als /wordzehen/ bezeichnet wird. In den Texten tritt der/dämos/dem Palast gegenüber korporativ als juristische Person auf, sodaß man keinen Einblick in seine innere Organisation gewinnt. Titel wie ko-to-ne-ta /ktoinetäs/105 und ko-tone-we /ktoinēwes/ bleiben ohne Profil. Nur die /ktoinohokhoi/ werden faßbar als Organe einer kommunalen Selbstverwaltung ${ }^{106}$.

Ausgehend von einer solchen Deutung der Texte, kann man ke-ke-mena ko-to-na als /kekesmenā kt./ auffassen, "abgeschnittenes Grundstück», "Parzelle des unter den /dämos/-Mitgliedern aufgeteilten ( = aufgeschnittenen) Bodens ( $^{107}$, oder als /kekhemenā kt./im Sinne von "(von Palastleuten) leer gelassener = unbesiedelter Grundbesitz«. Es ist

sondern sich stärker aufgliederte und sich dabei mit den privaten Eigentumsrechten und Besitztiteln in den einzelnen Gemeinden auseinanderzusetzen hatte" (in E. LIPIŃSKI [Hg.], State and Temple Economy in the Ancient Near East, Leuven 1979, Bd. II, S. 456).

100 Einzeluntersuchungen zu den diversen Titeln und ihren Trägern sind Legion, eine zusammenfassende Studie fehlt. Die Daten sind gesammelt bei M. LINDGREN, op. cit. (Fn. 24). Siehe ferner die in Fn. 2 angeführte Literatur und L. R. PALMER, Interpretation of Mycenaean Greek Texts, Oxford 1963.

101 J. T. KILLEN, in Colloquium Mycenaeum (s. Fn. 7), S. 177.

102 J. T. Killen, ibid.; Deger-Jalkotzy, E-QE-TA (s. Fn. 10), S. 72.

103 Siehe oben S. 45.

104 Siehe unsere Fn. 73.

105 Dazu S. Deger-Jalkotzy, op. cit. (Fn. 15), S. 96, nach A. Leukart, in H. Rix (Hg.), Flexion und Wortbildung (Fachtagung Regensburg 1973), Regensburg 1975, S. 180, 183-189.

106 Siehe unsere Fn. 49.

107 Dieser Interpretation gab ich in Res Mycenaeae (siehe Fn. 15), S. 95f. mit Fn. 38 den Vorzug. Zum graphischen Problem des /-sm-/ siehe aber oben, Fn. 38. 
aber gut möglich, daß /kekhemenā kt./ die Grundbedeutung »leer lassen, leer machen « in einem ganz anderen Sinn enthält.

Abschließend sei nochmals betont, wie begrenzt die Evidenz der mykenischen Texte ist. Was man durch die interne Kombination ihrer Aussagen gewinnen kann, ist heute erarbeitet. Trotzdem bleibt das System, das hinter diesen lakonischen Einzeldaten steht, bruchstückhaft und kann eigentlich nur durch Extrapolation erschlossen werden. Dabei gewinnt heute in zunehmendem Maß die Erkenntnis Raum, daß Analogien für die mykenischen Verhältnissen viel eher in den Staaten des zeitgleichen Alten Orients zu suchen sind als in Systemen, die zeitlich weit von der mykenischen Periode getrennt sind. Sie sind aber auch vorzuziehen einer Projektion der griechischen Verhältnisse des 1. Jahrtausend v.Chr. zurück auf die mykenische Periode. Disputabel ist natürlich die Frage, welche Staaten des Alten Orients die besten Parallelen bieten. Das generelle System, das ich als "Palaststaat" bezeichnen möchte, ist überall zu finden. Engere Analogien sind vielleicht bei den Hethitern zu erwarten, wo die Ausgangssituation einer indogermanischen Oberschichte über einem nichtindogermanischen Substrat zu vergleichbaren Resultaten geführt haben mochte. Noch enger aber sehe ich die Analogien zu Ugarit durch die Kleinheit des Territoriums und die Lage am Meer. Ähnlich wie dort ${ }^{108}$, dürfte auch für die mykenischen Voraussetzungen das Palastsystem von vornherein ungeeignet und darum höchst verwundbar gewesen sein. Die große Belastung der Bevölkerung mit Leistungen für den wuchernden Palastsektor ${ }^{109}$, das im Vergleich zu den Anforderungen des Palaststaates viel zu kleine Territorium, woraus eine extreme Ausbeutung der geringen Ressourcen und vor allem des Ackerbodens resultierte, und dazu die prekäre Lage zwischen der Politik der Großmächte der Zeit mußten auf Dauer zu einer Schwächung führen, die Ugarit so wie die mykenischen Palaststaaten zu einer leichten Beute für die fatalen Angriffe machten, denen die östliche Mittelmeerwelt um 1200 v.Chr. ausgesetzt war ${ }^{110}$.

S. Deger-Jalkotzy

Institut für Alte Geschichte und Altertumskunde

Residenzplatz 1

A-5020 Salzburg

${ }^{108}$ Zur Information dienen vor allem die beiden, Fn. 57 und 99 angeführten Studien von M. HeLtZER. Dort auch ausführliche Literaturangaben.

109 Siehe oben S. 49.

110 Während der Drucklegung sind erschienen: P. H. Ilievski- L. Crepajac ( $\mathrm{Hg}$.), Tractata Mycenaea, Skopje 1987 (unsere Fn. 25, 42, 92); R. HäGg-N. Marinatos (Hg.), The Function of the Minoan Palaces, Stockholm 1987 (unsere Fn. 14); E. LÉvy (Hg.), Le système palatial en Orient, en Grèce et à Rome, Strasbourg 1987 (Fn. 12). 